extensive sampling of the surrounding adipose tissue away from the tumour will be discussed.

\section{TUMOUR TO TUMOUR METASTASIS IN A PERIPHERAL NERVE SHEATH TUMOUR}

\section{J. Turchini, J. Chen}

Royal North Shore Hospital, St Leonards, NSW, Australia

Case Presentation: A 68-year-old woman with known metastatic breast cancer developed an ataxic gait, lower limb weakness and urinary retention over the course of one week. A magnetic resonance imaging (MRI) scan was performed which showed a T10/T11 intradural tumour. She was subsequently taken to theatre for decompression and resection of the tumour. The bulk of tumour was composed of densely packed bland tapered spindle cells arranged in fascicles. There were no features of schwannoma identified. Admixed within the tumour were cords and single cells of metastatic poorly differentiated adenocarcinoma with mucin droplets. Immunohistochemistry within the spindle cells confirmed the presence of a peripheral nerve sheath tumour, and within the epithelioid cells confirmed the presence of metastatic breast carcinoma.

Discussion: Tumour to tumour metastasis (TTM) is a well described phenomenon. It involves lung, breast and gastrointestinal tract malignancies as the most common donors. Meningiomas and schwannomas are common receptive tumours. However in this case it is difficult to ascertain if the receptive tumour was a neurofibroma or a schwannoma. Distinguishing a TTM from a sarcomatous biphasic malignancy is an important consideration, especially in the setting of a primary diagnosis.

\section{GASTRIC-TYPE ADENOCARCINOMA OF THE CERVIX: A CASE REPORT}

Melisa Vazquez, Gayanie Ratnayake

Department of Anatomical Pathology, The Royal Women's

Hospital, Sydney, NSW, Australia

Gastric-type adenocarcinoma of the cervix (GAC) is an uncommon and malignant tumour to human papilloma virus infection. Patients often present at an advanced stage and have a worse prognosis than those with usual type endocervical adenocarcinoma. We describe a case of stage IV GAC diagnosed in our institution. This case report illustrates the distinct morphological and immunohistochemical profile of these tumours and serves as basis for a brief discussion of prognosis and associated syndromes.

\section{CLEAR CELL MENINGIOMA: A CASE REPORT WITH REVIEW OF THE LITERATURE}

Runjin Wang ${ }^{1}$, Darweesh Al-Khawaja ${ }^{2}$,

Concepcion Chiribao-Negri

${ }^{1}$ Department of Anatomical Pathology, Nepean and Blue

Mountains Pathology Service, and ${ }^{2}$ Department of

Neurosurgery, Nepean Hospital, NSW, Australia

Clear cell meningioma (CCM) is a rare, aggressive variant of meningioma, usually affecting young females with frequent recurrence and CNS metastasis. Herein, we report a case of intracranial CCM in a 39-year-old female. The patient presented with severe headache and facial pain. MRI demonstrated a large extra-axial lobulated mass in the right cerebellopontine angle cistern with post-contrast enhancement. Histopathology of the tumour showed typical features of CCM. The tumour cells were PAS positive, diastase sensitive and immunopositive for epithelial membrane antigen (EMA). This case illustrates typical features of a rare variant of meningioma. It also highlights the importance of incorporating immunohistochemistry in the diagnostic workup to exclude CCM mimics, each having distinctive biological behaviour and prognostic outcome.

\section{HEPATOCELLULAR MALIGNANT NEOPLASM NOS: CASE REPORTS OF A NEW PROVISIONAL ENTITY}

Jennifer Wang, Nicole Graf, Susan Arbuckle

Histopathology Department, The Children's Hospital at

Westmead, Sydney, NSW, Australia

Hepatoblastoma (HB) is the most common paediatric liver malignancy, occurring almost exclusively in infancy. Paediatric hepatocellular carcinoma (HCC) is much less common. The International Pediatric Liver Tumors Consensus Classification (2014) suggested a provisional entity, 'hepatocellular malignant neoplasm, NOS' (previously known as 'transitional cell liver tumour') for difficult to classify tumours with overlapping morphological features, but generally very high alpha-fetoprotein levels at diagnosis. These tumours occur in a relatively older age group than classic $\mathrm{HB}$, and are associated with poorer prognosis and response to treatment than expected for HB. We present two such cases of multifocal liver tumours, in an 8-year-old girl and a 7-year-old boy, both with no evidence of underlying liver disease. These tumours both showed histological features favouring HCC, including large tumour cells with a single prominent nucleolus and intranuclear pseudoinclusions, arranged mostly in trabeculae. There was no associated cirrhosis. Focal extramedullary haemopoiesis was present in case 2 , but seen only at resection (not on biopsy). Both had AFP levels higher than expected for HCC that decreased following chemotherapy, as expected with HB.

Conclusion: Hepatocellular malignant neoplasm (NOS) has been suggested as a provisional category for paediatric tumours not readily classifiable as either HB or HCC.

\section{PARATHYROID LIPOADENOMA WITH BROWN FAT RICH FEATURES: CASE REPORT}

Morgan Wang, Simon Ryan, Marek Havlat
Western Diagnostic Pathology, WA, Australia

A 52-year-old man with primary hyperparathyroidism underwent a right superior parathyroidectomy. Pathological examination confirmed the presence of a lipoadenoma with a brown fat-rich stroma. This is a new, previously undescribed variant which expands the morphological range of patterns for this rare entity.

Review and discussion: Parathyroid lipoadenomas are a rare variant of parathyroid adenomas, with $\sim 35$ reported cases in the literature. Case series from Mayo Clinic ${ }^{1}$ and The University of Pittsburg Medical Centre ${ }^{2}$ have shown that they behave similar 